\title{
Laryngotracheal edema due to thermal injury: A complication after thyroidectomy in children
}

\author{
Pinar Kendigelen, M.D. ${ }^{a}$; Ayse C. Tutuncu, M.D. Assoc. Prof. ${ }^{a}$; Gulruh Ashyralyyeva, M.D. ${ }^{a}$; \\ Senol Emre, M.D. ${ }^{b}$, Seval Urkmez, M.D. ${ }^{a}$, Tughan Utku, M.D. Assoc. Prof. ${ }^{a}$ and Guner Kaya, M.D., Prof. ${ }^{a}$
}

\begin{abstract}
Postoperative respiratory insufficiency is a serious complication of total thyroidectomies which can be multifactorial, especially in children. We report two siblings who had undergone thyroidectomy with subsequent respiratory distress. Electrothermal bipolar and harmonic scalpel were used during thyroid dissections. Both patients had early postoperative respiratory problems. The older one suffered from mild respiratory distress for 24 hours and then he spontaneously recovered. The younger one was extubated but then she had serious stridor accompanied with abdominal and intercostal retractions. She was re-intubated and admitted to ICU for mechanical ventilatory support, where she stayed for 14 days due to multiple failed extubation attempts.

The symptoms were more severe in the younger child probably due to softer tracheal wall and weaker tracheal cartilages. We should keep in mind the probable postoperative respiratory complications due to thermal injury or inappropriate surgical technique after thyroid surgeries.

Key words: thyroidectomy, child, laryngotracheal edema.
\end{abstract}

http:/ /dx.doi.org/10.5546/aap.2017.eng.e31

\section{INTRODUCTION}

The thyroid is a butterfly-shaped gland which is located in front of the trachea. Thyroid cancer is the third most common solid tumor malignancy and the most common endocrine malignancy in children. Children with a family history of multiple endocrine neoplasia (MEN) are at high risk of developing medullary thyroid carcinoma (MTC). ${ }^{1}$ Prophylactic total thyroidectomy is recommended in children whose genetic screen shows the MEN2 gene mutation to prevent the occurrence of carcinoma. ${ }^{2}$

a. Department of Anesthesiology and Intensive Care, Cerrahpasa Medical School, Istanbul University, Istanbul, Turkiye.

b. Department of Pediatric Surgery, Cerrahpasa Medical Faculty, Istanbul University, Istanbul, Turkiye.

E-mail address:

Pinar Kendigelen, M.D.: pinarken@gmail.com

Funding: None.

Conflict of interest: None.

Received: 5-11-2016

Accepted: 8-11-2016
Postoperative respiratory insufficiency can be a very serious complication of thyroid surgeries, especially in children. We present two children with respiratory insufficiencies, one short and one long term, after total thyroidectomy.

\section{CASE REPORT}

We report two siblings, a 9-year-old boy and a 3.5-year-old girl, both known with MEN2b due to p.c618s RET proto-oncogene mutation. They had undergone prophylactic thyroid removal surgery to prevent MTC. Both had early postoperative respiratory insufficiency due to upper airway obstruction.

The siblings had a strong family history of MEN2B. Their grandmother underwent bilateral total thyroidectomy in 1996 and histopathology confirmed MTC. Father's genetic testing revealed the p.c618s RET proto-oncogene mutation. Thus, the patients underwent genetic testing. Heterozygote p.c618s mutation was identified in both cases. Patients' thyroid function test (TFT), thyroglobulin (TG), calcitonin and parathyroid hormone (PTH) results were normal. The girl's thyroid gland ultrasonography was normal but the boy had a $2.5 \mathrm{~mm}$ nodule. The endocrine multidisciplinary committee decided to perform prophylactic thyroidectomy.

The same anesthetic management was performed in both cases. Propofol $(2 \mathrm{mg} / \mathrm{kg})$, fentanyl $(1 \mu \mathrm{g} / \mathrm{kg})$ and rocuronium $(0.6 \mathrm{mg} / \mathrm{kg})$ were administered for induction. The patients were intubated with un-cuffed tubes (No: 6.5 and 5.0 Rüsch Flex for the boy and the girl, respectively). Patients received dexamethasone $(0.1 \mathrm{mg} / \mathrm{kg})$. The duration of surgeries was approximately 2 hours. The neuromuscular blockade was reversed with neostigmine $(0.02 \mathrm{mg} / \mathrm{kg})$ after premedication with atropine (0.01 mg/kg).

The thyroidectomy was performed with the same surgical method by the same surgical team. Electro-thermal bipolar vessel sealing system (LigaSure $^{\mathrm{TM}}$, Covidien-Medtronic) and harmonic scalpel (Harmonic ${ }^{\circledR}$, Ethicon Endo-Surgery) were used during dissection of thyroids from 
the trachea. In both cases, the right laryngeal recurrent nerve had been identified, but the left bundle of the nerve hadn't been in girl's case.

In the first case, the boy was waking up with cough after extubation. He had minimal stridor and intercostal retractions as well. Nebulized budesonide and nebulized racemic adrenaline were administrated. He was observed in postoperative care unit for 3 hours and then transferred to the ward. He suffered from respiratory distress for 24 hours and then recovered. In the second case, the girl had severe stridor as well as abdominal and intercostal retractions after extubation. An additional dose of IV dexamethasone $(0.1 \mathrm{mg} / \mathrm{kg})$, nebulized budesonide, nebulized racemic adrenaline and IV magnesium were administrated. We decided to re-intubate her when $\mathrm{SpO}_{2}$ dropped to $60 \%$ due to obstructive respiratory insufficiency. First, we observed vocal cords under direct laryngoscopy. The vocal cords were mobile, however laryngeal edema was presented. So she was re-intubated with a narrower un-cuffed tube (no: 4 Rüsch Flex) than the previous one and then transferred to ICU under sedation and mechanical ventilation.

She remained dependent on mechanical ventilatory support for 14 days. Multiple extubation trials were performed but she could not sustain adequate spontaneous breathing due to airway edema. She suffered from stridor and substernal retractions. Steroids and antibiotics were administered for two weeks. Levothyroxine $(25 \mu \mathrm{gr})$ was started upon pediatric endocrinologist's recommendation. The laboratory findings, including $\mathrm{Ca}^{++}$, were within normal limits. On the $8^{\text {th }}$ day of ICU stay bronchoscopy was performed and the mobile vocal cords and semi-collapsed trachea at subglottic level during inspiration were observed during spontaneous breathing. Thus, diagnosis of tracheomalacia was established. On the $14^{\text {th }}$ day of ICU stay, a second bronchoscopy was performed and evaluated by both otorhinolaryngology and pediatric surgeons. The patient was orotracheally intubated, awake with spontaneous breathing and sedated with midazolam $(0.2 \mathrm{mg} / \mathrm{kg})$. Then she was extubated to observe her clinical status before making a decision about performing a tracheostomy. She was closely observed for about two hours and had minimal substernal retractions and breathing distress. The arterial $\mathrm{PaO}_{2}$ and $\mathrm{PCO}_{2}$ levels were monitored during this period and were within normal ranges. She was transferred to ICU after two hours of spontaneous breathing and minimal respiratory distress. The patient was discharged to the ward after 24 hours.

Diffuse thyroid hyperplasia was histologically confirmed in both cases. In addition, reactive hyperplasia in two lymph nodes and focal $C$ cell hyperplasia were confirmed in the first case. Both patients had been consulted by pediatric oncology and endocrinology teams and their future treatment will be discussed in thyroid multidisciplinary committee.

\section{DISCUSSION}

Laryngotracheal surgery requires attentive approach and enough experience to prevent risk of postoperative edema. The serious complications as laryngeal edema and respiratory distress are rarely seen. The reasons of postoperative respiratory obstruction following thyroidectomy are laryngotracheal edema, tracheal collapse and vocal cord paralysis. ${ }^{3}$

Laryngotracheal edema in subglottic area, which is the narrowest part of airway in children, is one of the most frequent reasons of postoperative airway obstruction. The edema may also be developed in supraglottic area due to dissection of the upper thyroid cartilage. It usually causes mild symptoms but can be life threatening. The etiology of edema is mainly the surgical trauma, however intubation difficulties contribute as well. ${ }^{4}$ In our patients, especially in the girl, exaggerated respiratory obstruction was observed due to the laryngotracheal edema. The symptoms were more severe in the younger child because of the soft tracheal wall and weak tracheal cartilages. ${ }^{5}$ We observed tracheomalacia and tracheal collapse during bronchoscopy performed on the $8^{\text {th }}$ postoperative day. The brother's symptoms improved faster than sister's based on age-related tracheal maturity.

In these cases, intubations were performed with properly sized un-cuffed tubes and no postoperative vocal cord paralysis was observed so these made us think about other possible reasons of laryngotracheal edema. The thyroid tissue was removed completely due to high risk of malignancy. The benefits of using Harmonic scalpel and electrosurgical instruments are short resection time, good hemostasis but they have as possible risk of thermal injury in surrounding tissue. ${ }^{6}$ The mechanism of action of surgical instruments is different. The electrosurgical instrument use electric current but Harmonic scalpel use ultrasonic vibration. Despite of less formation of heat by Harmonic scalpel, there is 
still having a risk of thermal injury. Some tissues may be more affected as in our case so; it may increase the risk of thermal injury. Neither visible damage nor skin burn were observed in surgical site. The black-brown appearance was occurred due to instruments, wich were used to control coagulation during dissection. The both Harmonic Scalpel and Electro-thermal bipolar vessel sealing system were used in our cases. Thus, thermal injury due to surgery may be the main reason behind laryngotracheal edema, which can explain the early postoperative symptoms in these cases. Furthermore, an inappropriate surgical technique in this anatomical region can cause postoperative edema. The thermal injuries can be avoided by using accurate technic in this type of surgeries. The surgeon's experience in laryngotracheal operation is very important, especially in pediatric patients.

In summary, children have smaller airway diameters and weaker laryngeal structures. Thus, postoperative edema can generate obstruction more easily. Furthermore, postoperative edema can be occurred separately in several laryngeal structures. This singly edema of laryngeal structures may not lead to serious clinic but together they can generate obstruction. The surgical instruments should be chosen carefully in appropriate tissues due to prevent risk of complications. We should be well prepared for the probable respiratory complications due to thermal injury or inappropriate surgical technique after thyroid surgery in children.

\section{REFERENCES}

1. Diesen DL, Skinner MA. Pediatric thyroid cancer. Semin Pediatr Surg 2012;21(1):44-50.

2. Lallier M, St-Vil D, Giroux M, Huot C, et al. Prophylactic thyroidectomy for medullary thyroid carcinoma in gene carriers of MEN2 syndrome. J Pediatr Surg 1998;33(6):846-8.

3. Wade JS. Cecil Joll Lecture, 1979. Respiratory obstruction in thyroid surgery. Ann R Coll Surg Engl 1980;62(1):15-24.

4. Martis C. Athanassiades S. Post-Thyroidectomy Larengeal Edema. A Survey of Fifty-Four Cases. Am J Surg 1971;122(1): 58-60.

5. Breuer C, Tuggle C, Solomon D, Sosa JA. Pediatric Thyroid Disease: When is Surgery Necessary, and Who Should be Operating on Our Children? J Clin Res Pediatr Endocrinol 2013;5(Suppl 1):79-85.

6. Cordón C, Fajardo, Ramirez J, Herrera MF. Randomized, prospective, parallel group study comparing the Harmonic Scalpel to electrocautery in thyroidectomy. Surgery 2005;137(3):337-41. 